\title{
Effects of the HDAC inhibitor CG2 in combination with irinotecan, 5-fluorouracil, or oxaliplatin on HCT116 colon cancer cells and xenografts
}

\author{
YOUNG-SOON NA ${ }^{1}$, SEUNG-MI KIM ${ }^{1}$, KYUNG-AH JUNG ${ }^{1}$, SOO-JIN YANG ${ }^{1}$, \\ YONG SANG HONG ${ }^{2}$, MIN-HEE RYU ${ }^{2}$, SEONGGU RO ${ }^{1,4}$, DONG-HYUNG CHO ${ }^{1}$, \\ JIN CHEON KIM ${ }^{1,3}$, DONG-HOON JIN ${ }^{1}$, JUNG SHIN LEE ${ }^{1,2}$ and TAE WON KIM ${ }^{1,2}$ \\ ${ }^{1}$ Institute for Innovate Cancer Research, Departments of ${ }^{2}$ Oncology, and ${ }^{3}$ Surgery, Asan Medical Center, \\ University of Ulsan College of Medicine; ${ }^{4}$ CrystalGenomics, Inc., Seoul, Republic of Korea
}

Received August 3, 2010; Accepted September 24, 2010

DOI: 10.3892/or_00001012

\begin{abstract}
Chemotherapies for colon cancer have recently advanced. However, there is still a need to develop agents and identify effective regimens for better treatments of colon cancer. Histone deacetylase inhibitors (HDACIs) have shown potential as anti-cancer agents. We investigated the anti-tumor effects of CG2 (an HDACI) in combination with irinotecan, 5-FU, or oxaliplatin. Combinations of CG2 with SN38 (the active form of irinotecan), 5FU, or oxaliplatin were more effective than the agents alone when used to inhibit the growth of HCT116 cells. The protein expressions of acetyl-H3, p21, caspase-3, -8, and -9, PARP, and XIAP were affected in a time- and dose-dependent manner in HCT116 cells treated with the CG2 alone or combined CG2 and SN-38. In HCT116 xenografts, the HDACI CG2 in combination with irinotecan dramatically inhibited tumor growth without showing additive toxicity. These data indicate that CG2 together with irinotecan is a promising combination novel treatment for colon cancer.
\end{abstract}

\section{Introduction}

Over the past several decades, significant therapeutic improvements have been achieved in the treatment of metastatic colorectal cancer (mCRC) patients, including the use of 5-fluorouracil (5-FU), irinotecan, or oxaliplatin. 5-FU, an antimetabolite, inhibits thymidylate synthase (TS) (1). Irinotecan is an inhibitor of topoisomerase I and is activated

Correspondence to: Dr Tae Won Kim, Department of Oncology, Institute for Innovate Cancer Research, Asan Medical Center, University of Ulsan College of Medicine, 86 Asan Byungwon Gil, Songpa-gu, Seoul 138-736, Republic of Korea

E-mail: twkimmd@amc.seoul.kr

Key words: histone deacetylase inhibitors, CG2, irinotecan, 5-FU, oxaliplatin, colon cancer to the compound $\mathrm{SN}-38$ by the carboxylesterase of mammalian tissue (2). Oxaliplatin is a platinum alkylator of DNA (3).

Recent studies have suggested that survival outcomes can possibly be further improved by the addition of cetuximab, panitumumab, or bevacizumab to chemotherapy regimes (4). However, no effective agents are available if these drugs fail, and a clear need for new therapeutic approaches is apparent.

Histone deacetylase inhibitors (HDACIs) induce apoptosis, cell cycle arrest, and differentiation of tumor cells (5). Several HDACIs including trichostatin A (TSA), SAHA, PXD101, LAQ-824, and LBH589, have been investigated for use in the treatment of various hematologic malignancies and solid tumors, including colon cancer.

Several HDACs are overexpressed in colon cancer, and may contribute to cancer progression by epigenomic repression of tumor suppressor genes, or by mediating hypoacetylation and functional modification of non-histone substrates. The anti-tumor action of HDACIs lies in the reversal of such effects. HDACIs have been reported to show anti-tumor effects when used as single agents or in combination with other drugs, both in vitro and in vivo (6,7). CG2 (CrystalGenomics, Seoul, Korea; Fig. 1) is a novel hydroxamate-based panHDACI $(8,9)$ that is under clinical development. We previously reported that the use of CG2 in combination with FOLFIRI (5-FU/leucovorin/irinotecan) was associated with synergistic effects and a markedly higher response rate compared with SAHA in in vitro tissue culture of CRC (8). In the present study, using both in vitro and in vivo assays, we evaluated the effects of CG2 in combination with irinotecan, 5-FU, or oxaliplatin, agents that are clinically used to treat colon cancer. A combination of CG2 and irinotecan dramatically inhibited tumor growth, without development of additive toxicity, compared with other combinations tested. These data indicate that CG2 combined with irinotecan is a promising therapeutic combination for treatment of colon cancer.

\section{Materials and methods}

Cell line, chemicals, and antibodies. The human colon cancer cell line HCT116 was purchased from the American Type 


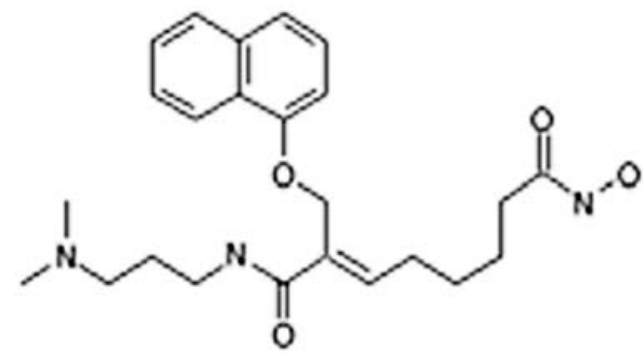

Figure 1. The chemical structure of CG2.

Culture Collection (Manassas, VA). CG2 has been developed by CrystalGenomics. Irinotecan, oxaliplatin, SN-38, and 5-FU were obtained from Pfizer Korea, Sanofi Aventis Korea, Hanmi Pharmaceuticals, and Choongwae Pharmaceuticals Corporation (all of Seoul, Korea), respectively. Primary antibodies against acetyl-H3; H3; PARP; caspases-3, -8, and -9; p21; XIAP; and $\beta$-actin (Cell Signaling, Danvers, MA) were used in Western blot analyses.

Cell viability assay. Cell viability was determined using the CellTiter 96 aqueous non-radioactive cell proliferation assay (Promega, Madison, WI) according to the manufacturer's instructions. The experiments were performed in duplicate on three independent occasions. Cells were exposed to single agents or combinations of two agents for $48 \mathrm{~h}$, and sequential treatment of HCT116 cells involved exposure to the HDACI for $24 \mathrm{~h}$ followed by addition of SN38, 5-FU, or oxaliplatin for $24 \mathrm{~h}$, or vice versa. Each cell viability curve was plotted as the relative change with respect to untreated cells and $\mathrm{IC}_{50}$ values were calculated using GraphPad Prism software (Graphpad Software Inc., San Diego, CA).

Xenograft model. Tumors were established by subcutaneous injection of $5 \times 10^{6}$ HCT116 cells into the left flank of athymic nude mice (SLC, Hamamatsu, Japan). When the subcutaneous tumors attained a volume of $100 \mathrm{~mm}^{3}$ (day 0 ), test agents were injected intraperitoneally (i.p.). CG2 and 5-FU were given at doses of 20 and $10 \mathrm{mg} / \mathrm{kg}$, respectively, for 5 days, followed by 2 days without treatment, each week, for three cycles. Irinotecan and oxaliplatin were injected at doses of 50 and $10 \mathrm{mg} / \mathrm{kg}$, respectively, once weekly over three cycles. Combinations of CG2 with other agents were administered by injection of CG2 in the morning followed by irinotecan, oxaliplatin, or 5-FU in the afternoon, over three cycles. Tumor volume was calculated in $\mathrm{mm}^{3}$ as (length in $\mathrm{mm} \mathrm{x}$ width in $\left.\mathrm{mm}^{2}\right) / 2$. Body weight was also monitored. On days 4 and 11 , tumor specimens were collected for TUNEL analysis $4 \mathrm{~h}$ after injection of CG2, at the time of maximal level of acetylH3. The study was approved by our Institutional Animal Care and Use Committee (IACUC).

Preparation of tumor tissue extracts and TUNEL staining. Dissected tumors were homogenized in tissue lysis buffer [50 mmol/l Tris- $\mathrm{HCl}$ (pH 8.0), $150 \mathrm{mmol} / \mathrm{l} \mathrm{NaCl}, 0.02 \%$ sodium azaide, $0.1 \%$ SDS, $1 \%$ NP-40, $0.5 \%$ sodium deoxycholate, $10 \mu \mathrm{l} / \mathrm{ml}$ protease inhibitor mix]. Homogenates were centrifuged and analyzed by Western blotting. TUNEL staining
Table I. $\mathrm{IC}_{50}$ values of various agents acting on HCT116 cells.

\begin{tabular}{lc}
\hline Agent & $\mathrm{IC}_{50}$ \\
\hline CG2 & $0.55 \pm 0.22 \mu \mathrm{M}$ \\
SAHA & $1.04 \pm 0.31 \mu \mathrm{M}$ \\
SN38 & $2.57 \pm 0.85 \mathrm{nM}$ \\
5-FU & $6.16 \pm 1.27 \mu \mathrm{M}$ \\
Oxaliplatin & $1.24 \pm 0.93 \mu \mathrm{M}$ \\
\hline
\end{tabular}

The values shown are means \pm SD of data from three independent experiments.

of tumor tissues was performed using an In Situ Cell Death Detection kit (Roche-Applied Science, Indianapolis, IN) following the manufacturer's instructions. 4'-6-diamidino-2phenylindole (DAPI) was used for counterstaining. Three distinct high-power fields of each slide were analyzed under a fluorescence microscope. Signals were quantified using Image J software (National Institutes of Health, Bethesda, MD).

Statistical analysis. Data are expressed as means \pm SEM. Differences between test groups were analyzed by the MannWhitney test or the unpaired t-test using GraphPad InStat (Graphpad Software Inc.). P $<0.05$ were considered statistically significant.

\section{Results}

Growth-inhibitory activities of CG2, SAHA, oxaliplatin, and 5-FU, measured using the human colon cancer cell line HCT116. To investigate the growth-inhibitory capacity of the novel HDACI candidate CG2, and those of SAHA, oxaliplatin, and 5-FU, using HCT116 cells, we determined $\mathrm{IC}_{50}$ values after treatment of cells with inhibitors for $48 \mathrm{~h}$ (Table I). The $\mathrm{IC}_{50}$ value of SN38 when used to inhibit growth of HCT116 cells was $2.57 \mathrm{nM}$ in our previous study (10). The growthinhibitory activity of CG2 was similar to that of SAHA.

Cytotoxic effects of simultaneous or sequential exposure of HCT116 cells to various agents. To examine the cytotoxic effects mediated by simultaneous or sequential exposure to HDACI, on the one hand, and SN38, 5-FU, or oxaliplatin, on the other, cell proliferation assays were used. When HDACI and SN38 were combined, the extent of cytotoxicity was not dependent on the sequence of administration (Fig. 2A). However, sequential treatment of HCT116 cells with CG2 or SAHA followed by 5-FU resulted in a greater extent of cytotoxicity than was seen when the reverse sequence was used, being similar to that seen after simultaneous treatment (Fig. 2B). When HCT116 cells were treated with oxaliplatin followed by CG2 or SAHA, the cytotoxic effect was greater than when the reverse sequence was used (Fig. 2C). Therefore, the cytotoxic effects of HDACI, combined with 5-FU or oxaliplatin, were dependent on the sequence of agent administration, and simultaneous treatment with the HDACI and SN38, 5-FU, or oxaliplatin, were more effective than were the 
A

B
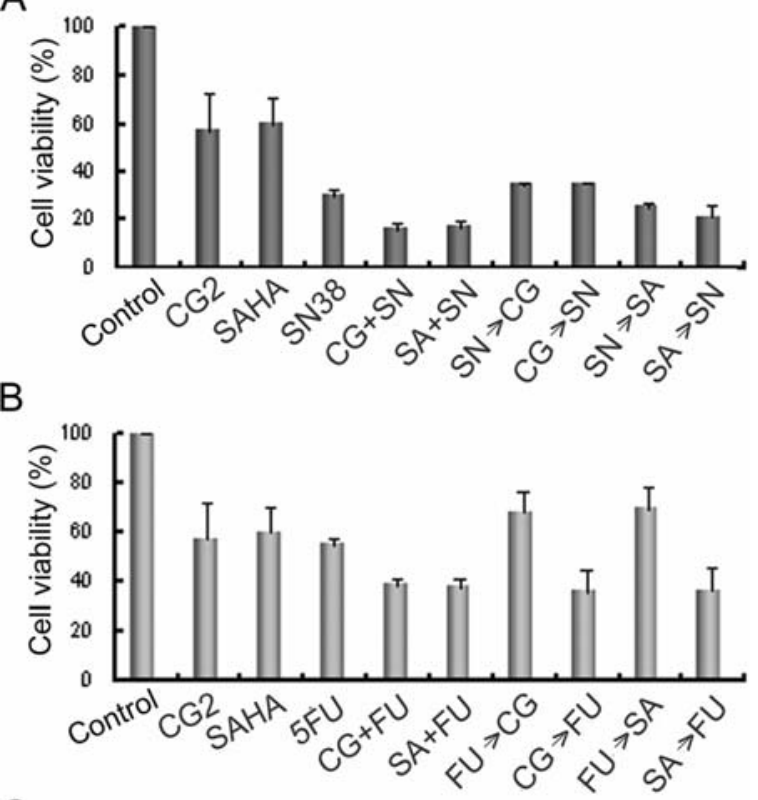

C

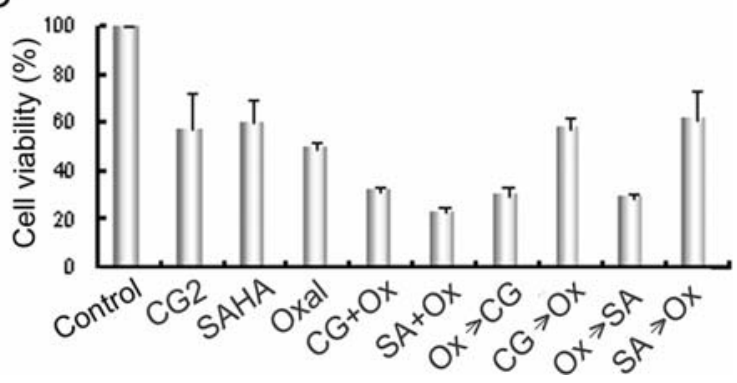

Figure 2. Growth inhibition after simultaneous or sequential exposure of HCT116 cells to various anti-cancer agents. HCT116 cells were treated with $1 \mu \mathrm{M}$ of CG2, SAHA, or oxaliplatin (C), or $50 \mathrm{nM} \mathrm{SN38}$ (A), or $7 \mu \mathrm{M}$ of 5-FU (B). CG, CG2; FU, 5FU; Oxal, oxaliplatin; SA, SAHA; SN, SN38.

single agents alone. These data indicate that administration sequence may be important in determining the extent of therapeutic synergy.

Effect of CG2 in combination with SN38 on protein expression in HCT116 cells. We performed Western blotting to examine the effects of single and combination treatments on the expression of acetyl-H3, H3, p21, XIAP, caspase-9, caspase-8, caspase-3, and PARP, in HCT116 cells, with respect to time after dosing (Fig. 3A) and dose level (Fig. 3B). As expected, acetyl-H3 was prominently induced in a time- and dosedependent manner by $\mathrm{CG} 2$ as well as by a CG2/SN38 combination. Also, p21 expression showed time- and dosedependent induction in HCT116 cells when CG2 and/or SN38 treatment was used. The level of the anti-apoptotic protein XIAP decreased in a time- and dose-dependent manner after CG2 treatment. Notably, XIAP expression was markedly reduced when CG2 was combined with SN38. The levels of PARP and caspase- $3,-8$, and -9 increased in a dose- and time-dependent manner after use of a combination of CG2 and SN38, but were only slightly increased, or remained unchanged, when SN38 alone was used. Thus, CG2 in combination with SN38 showed enhancement of the anti-apoptotic
A

$\frac{4 h}{c c^{2} \mathrm{sN}^{8} \mathrm{C}+{ }^{5} \mathrm{C} \mathrm{c} \mathrm{CGN}^{2} \mathrm{SN}^{8} \mathrm{C}+\mathrm{S}}$

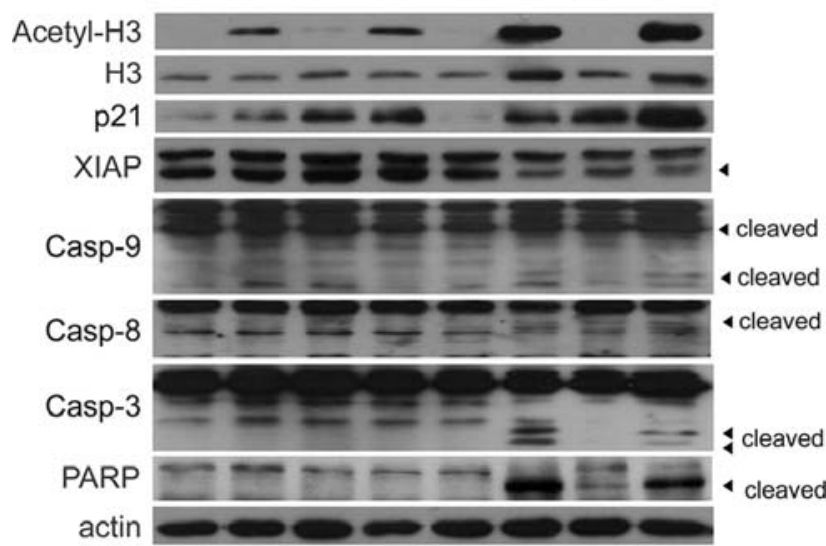

B
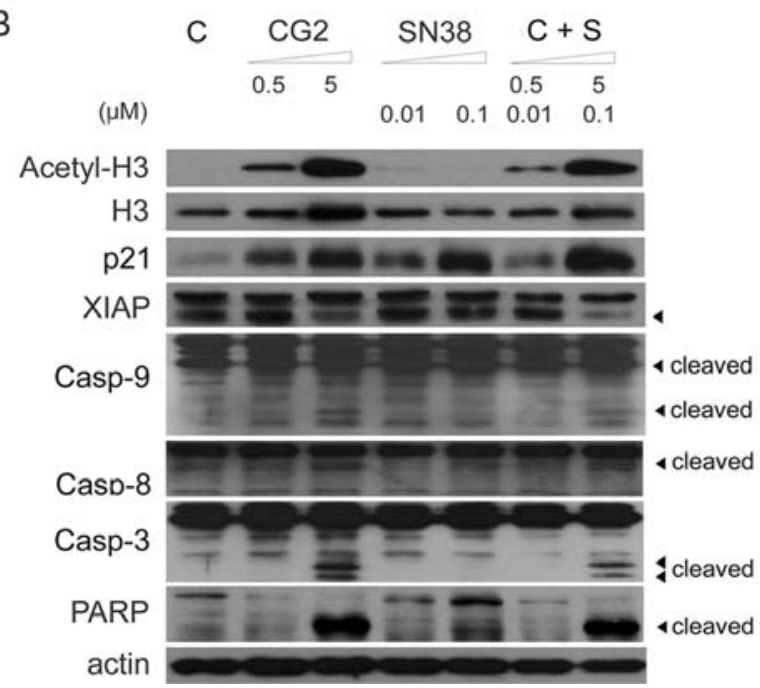

Figure 3. Effects of CG2 and/or SN38 on the expression of acetyl-H3, H3, p21, XIAP, PARP, and caspase-3, -8 , and -9 , with respect to (A) time since dosing and (B) dose level, in HCT116 cells. After treatment with $5 \mu \mathrm{M} \mathrm{CG} 2$ and/or $0.1 \mu \mathrm{M} \mathrm{SN} 38$ for $4 \mathrm{~h}$ or $24 \mathrm{~h}$, and with $0.5 \mu \mathrm{M}$ or $5 \mu \mathrm{M} \mathrm{CG} 2$ and/or $0.01 \mu \mathrm{M}$ or $0.1 \mu \mathrm{M} \mathrm{SN38}$ for $24 \mathrm{~h}$, protein expression levels in HCT116 cells were analyzed by Western blotting. $\mathrm{C}+\mathrm{S}, \mathrm{CG} 2+\mathrm{SN} 38$.

effect in HCT116 cells by significantly reducing the XIAP level.

Anti-tumor effects of CG2 in combination with irinotecan, 5-FU, or oxaliplatin, tested using HCT116 subcutaneous xenografts. To evaluate whether the combination of CG2 with irinotecan was synergistically active on HCT116 xenografts, we examined the anti-tumor effect of a combination of these agents, compared with either agent alone (Fig. 4A). Tumor growth inhibition values after treatment with CG2, irinotecan, and the combination were $10.4,11.8$, and $39.9 \%$, respectively, at day 3 , and $54.3,74.3$, and $87.8 \%$, respectively, at day $21(\mathrm{P}<0.05$ for the combination vs. CG2 or irinotecan alone). Therefore, administration of the combination afforded more effective tumor growth inhibition than single-agent CG2 or irinotecan, without loss of body weight.

Tumber et al reported that PXD101 in combination with 5-FU exhibited a synergistic anti-tumor effect in colon cancer 
A

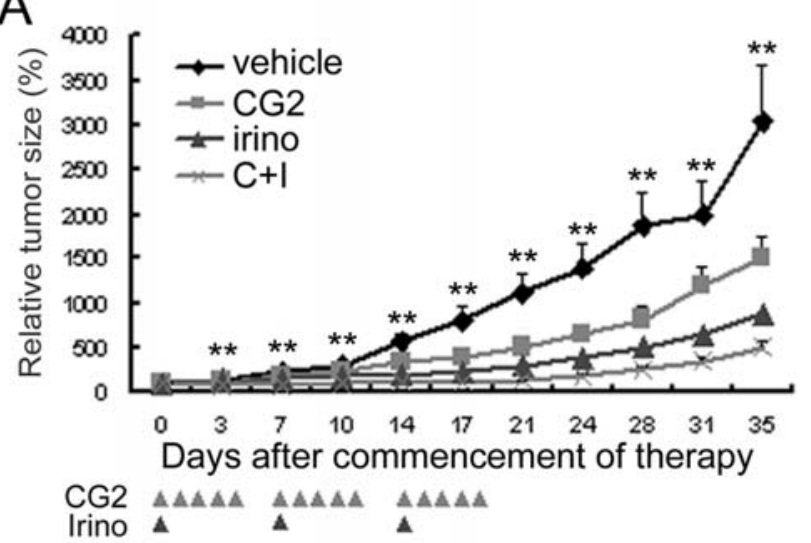

B

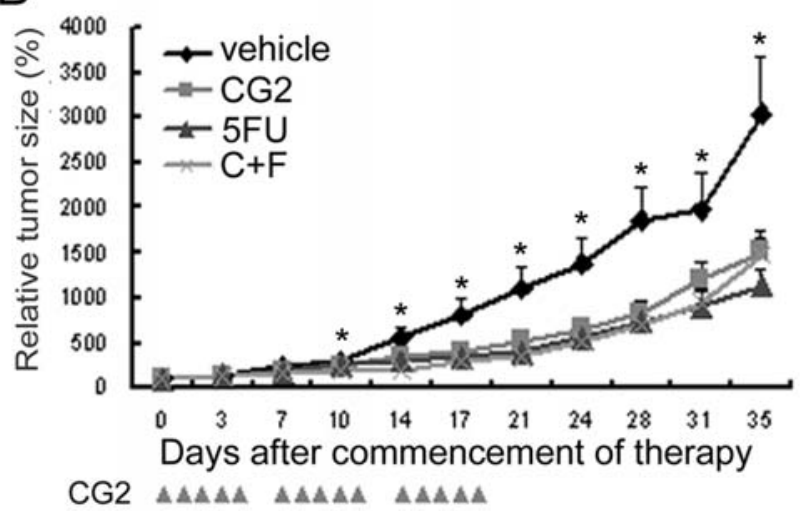

SFU AMAAM MAMAM MAMA

C

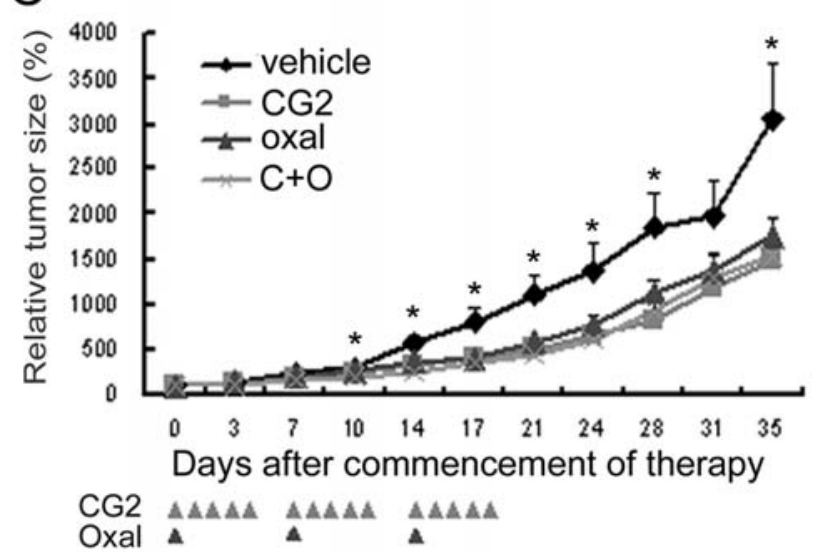

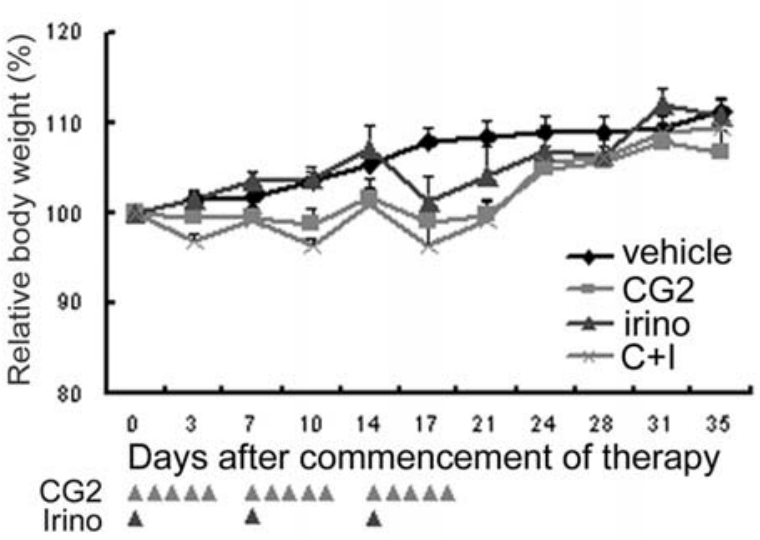

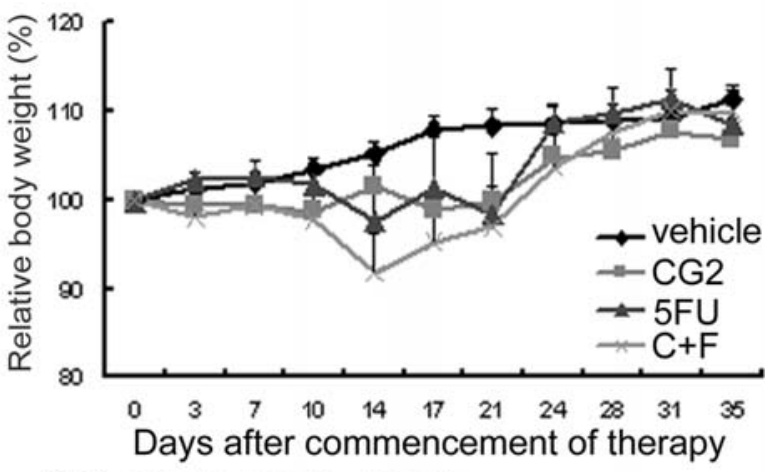

CG2 AMAA MAMA AMAMA

$5 F U$ MAMA MAMAM MAMAM

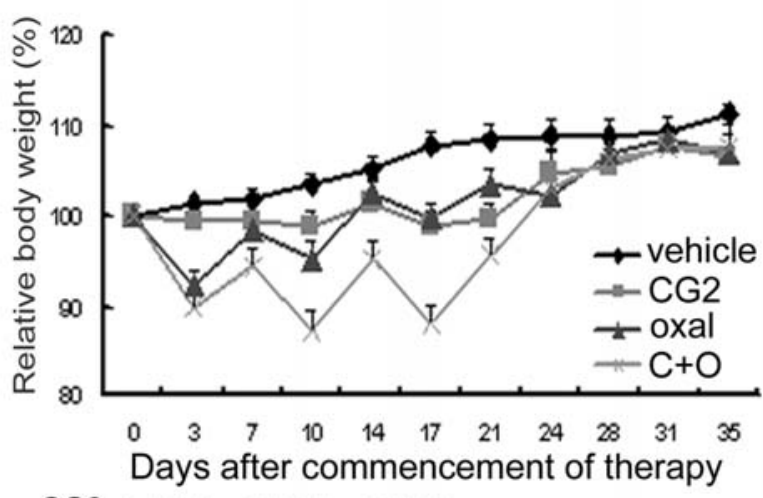

CG2 AMAM

Figure 4. Anti-tumor effects of CG2 in combination with irinotecan (A), 5-FU (B), or oxaliplatin (C), in the HCT116 xenograft model. (Vehicle, n=8; CG2, $\mathrm{n}=8$; irinotecan, $\mathrm{n}=6$; $\mathrm{C}+\mathrm{I}$ [CG2+irinotecan], $\mathrm{n}=8 ; 5 \mathrm{FU}, \mathrm{n}=6 ; \mathrm{C}+\mathrm{F}$ [CG2+5FU], $\mathrm{n}=8$; oxal (oxaliplatin), $\mathrm{n}=7 ; \mathrm{C}+\mathrm{O}$ [CG2+oxaliplatin], $\mathrm{n}=8$ ). The schedules of agent administration are indicated at the bottom of each graph. Comparisons of agent effects on tumor size were performed using the Mann-Whitney test; combination vs. vehicle, ${ }^{*} \mathrm{P}<0.05$; combination vs. individual agents, ${ }^{* *} \mathrm{P}<0.05$.

(11). Thus, we examined the anti-tumor effect of the CG2/ 5-FU combination using the HCT116 xenograft model (Fig. 4B). Based on the in vitro results, we chose to sequentially administer CG2 followed by 5-FU. However, the anti-tumor effect of the CG2/5-FU combination in HCT116 xenografts was similar to that of each agent alone.

Next, we evaluated the anti-tumor effect of a sequential CG2/oxaliplatin combination, again using the HCT116 xenograft model (Fig. 4C). This combination did not show an additive anti-tumor effect. Unlike that noted in vitro, the reverse sequence of agent administration did not result in any additive anti-tumor effect either (data not shown). Animals receiving the $\mathrm{CG} 2$ /oxaliplatin regimen lost a significant amount of weight over the three cycles of agent administration. Of the combinations tested, only CG2 combined with irinotecan showed an enhanced anti-tumor effect compared with that seen when the agents were used alone.

Analysis of protein expression in tumors arising from HCT116 xenografts after treatment with single agents, or with a 

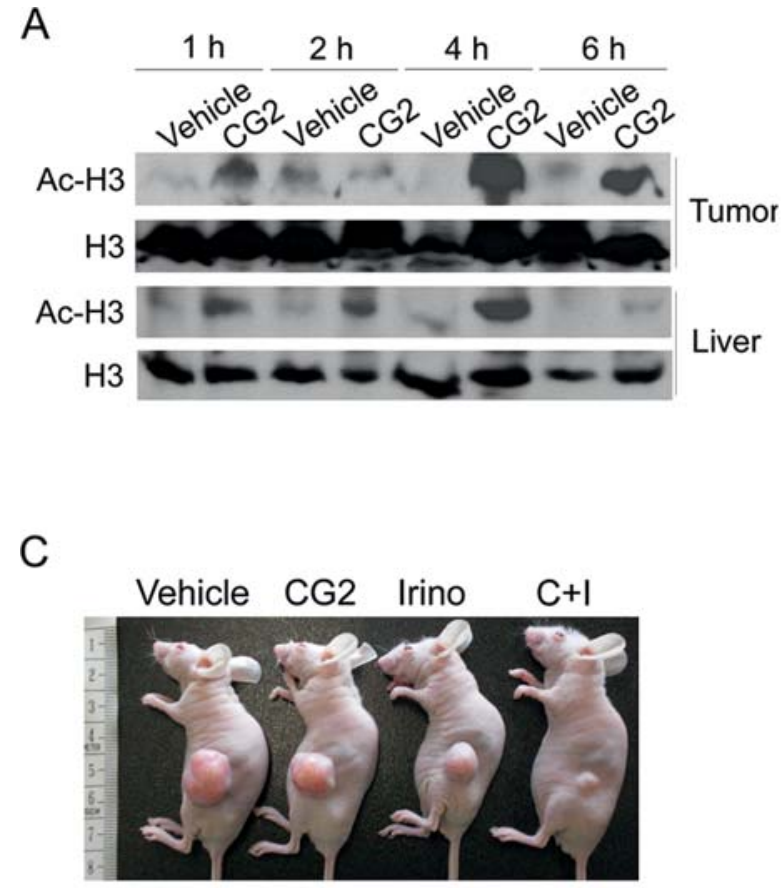

B
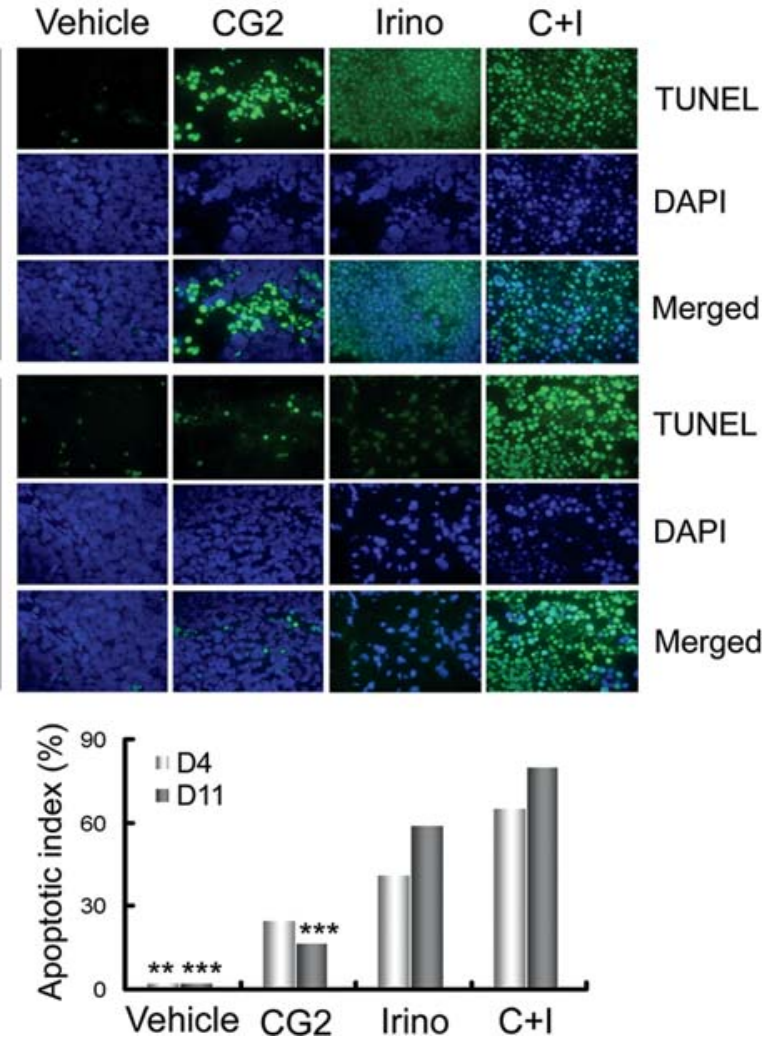

Figure 5. Analysis of tumor tissue in agent-treated HCT116 xenografts. (A) Expression of acetylated H3 in tumor and liver tissue at various times after administration of CG2. (B) TUNEL analysis on days 4 and 11 after treatment with various agents. Top: Representative tumor sections, x1,000. Bottom: Apoptotic index. Unpaired t-test; ${ }^{* *} \mathrm{P}<0.01$ vs. combination treatment; ${ }^{* * *} \mathrm{P}<0.001$ vs. combination treatment. (C) Representative HCT116 xenografts on day 35 after agent administration.

combination of CG2 and irinotecan. To measure the expression of acetylated $\mathrm{H} 3$ in tumor and liver after a single administration of CG2 to mice bearing HCT116 xenografts, tumor and liver samples were obtained 1, 2, 4, and $6 \mathrm{~h}$ after CG2 injection (Fig. 5A). The expression levels of acetyl-H3 in tumor and liver were markedly increased $4 \mathrm{~h}$ after treatment with CG2.

As a dramatic anti-tumor effect was seen when a combination of CG2 and irinotecan was administered, we performed a TUNEL assay on tumor tissue from xenografts treated with various agents to compare the level of apoptosis after administration of the CG2/irinotecan combination with that seen upon treatment with either agent alone (Fig. 5B). When agents were administered individually, the tumor cell apoptosis was over 3-fold that of the control vehicle group, but the CG2/irinotecan treatment resulted in elevation of apoptosis to a level in excess of 6-fold that of the control. Also, the extent of apoptosis on day 11 was slightly greater than seen on day 4. Tumor size in the combination therapy group, measured on day 35 , was dramatically decreased compared with that of other groups (Fig. 5C). Thus, the antitumor effect of the agent combination, as assessed by TUNEL, was better than that of either agent given alone.

\section{Discussion}

In the present study, we evaluated the anti-tumor effects of CG2 in combination with irinotecan, 5-FU, or oxaliplatin in colon cancer cells and in an in vivo model. CG2 in combination with irinotecan showed promising anti-tumor effects both in vitro and in vivo compared with other agent combinations.

A combined effect of TSA and irinotecan, acting on pancreatic and gastric cancer cells, has also been reported $(12,13)$. We previously demonstrated that PXD101 in combination with irinotecan dramatically enhanced the anti-tumor effect of each agent alone in in vitro and in vivo models of colon cancer (10). In line with these earlier findings, CG2 or SAHA in combination with SN-38 also had enhanced cytotoxic effects compared with those seen after administration of the individual agents. The increased potency of an HDACI combined with SN-38 might result from cooperative regulation of the DNA damage response by HDACI and SN-38 (14-16). Based on such results, a combination of SAHA with irinotecan has been tested in gastrointestinal cancer and recurrent glioblastoma (17).

Synergistic in vitro interactions between the HDACIs MS275 or SBHA and oxaliplatin have been reported (18). Our in vitro results also showed increased cytotoxicity when CG2 or SAHA were combined with oxaliplatin. A possible explanation for these observations is that an HDACI inhibits repair of oxaliplatin-induced DNA damage (19). We evaluated the effect of CG2 combined with oxaliplatin in HCT116 xenografts using a regimen based on our in vitro data. However, CG2 and oxaliplatin did not show administration sequencedependent effects on HCT116 xenograft regression (data not shown). In addition, CG2 in combination with oxaliplatin did 
not show any additive agent effect, in contrast to results from in vitro testing. Significant weight loss was observed when CG2 and oxaliplatin were administered together. Accordingly, any increase in the anti-tumor effect when CG2 is given together with oxaliplatin may not be useful in practice owing to the presence of various factors inducing toxicity in vivo.

Inhibition of TS expression following SAHA treatment might overcome 5-FU resistance in HCT116 cells $(20,21)$. In the present study, administration of an HDACI followed by 5 -FU seemed to be more effective than the reverse sequence. However, the anti-tumor effect seen after injection of $20 \mathrm{mg} / \mathrm{kg} \mathrm{CG} 2$ followed by $10 \mathrm{mg} / \mathrm{kg} 5-\mathrm{FU}$ in the HCT116 xenograft model was similar to that of each agent given alone. The absence of an increased anti-tumor effect may be attributable to the use of a low dose of 5-FU. In conclusion, our data indicate that CG2 in combination with irinotecan may be a valuable novel treatment for patients with colon cancer.

\section{Acknowledgements}

This study was supported by a grant from the Korea Health 21 R\&D Project, Ministry of Health and Welfare and Family Affairs, Republic of Korea (no. A062254).

\section{References}

1. Longley DB, Harkin DP and Johnston PG: 5-fluorouracil: mechanisms of action and clinical strategies. Nat Rev Cancer 3 : 330-338, 2003.

2. Teicher BA: Next generation topoisomerase I inhibitors: Rationale and biomarker strategies. Biochem Pharmacol 75 1262-1271, 2008.

3. Jennerwein MM, Eastman A and Khokhar A: Characterization of adducts produced in DNA by isomeric 1,2-diaminocyclohexaneplatinum (II) complexes. Chem Biol Interact 70: 39-49, 1989.

4. Kohne $\mathrm{CH}$ and Lenz HJ: Chemotherapy with targeted agents for the treatment of metastatic colorectal cancer. Oncologist 14: 478-488, 2009.

5. Glozak MA and Seto E: Histone deacetylases and cancer. Oncogene 26: 5420-5432, 2007.

6. Fazzone W, Wilson PM, Labonte MJ, Lenz HJ and Ladner RD Histone deacetylase inhibitors suppress thymidylate synthase gene expression and synergize with the fluoropyrimidines in colon cancer cells. Int J Cancer 125: 463-473, 2009.
7. Frew AJ, Lindemann RK, Martin BP, et al: Combination therapy of established cancer using a histone deacetylase inhibitor and a TRAIL receptor agonist. Proc Natl Acad Sci USA 105: 11317-11322, 2008.

8. Kim JC, Shin ES, Kim CW, et al: In vitro evaluation of histone deacetylase inhibitors as combination agents for colorectal cancer. Anticancer Res 29: 3027-3034, 2009.

9. Kim JC, Kim DD, Lee YM, et al: Evaluation of novel histone deacetylase inhibitors as therapeutic agents for colorectal adenocarcinomas compared to established regimens with the histoculture drug response assay. Int J Colorectal Dis 24: 209-218, 2009.

10. Na Y-S, Jung K-A, Kim S-M, et al: The histone deacetylase inhibitor PXD101 increases the efficacy of irinotecan in in vitro and in vivo colon cancer models. Cancer Chemother Pharmacol (In press).

11. Tumber A, Collins LS, Petersen KD, et al: The histone deacetylase inhibitor PXD101 synergises with 5-fluorouracil to inhibit colon cancer cell growth in vitro and in vivo. Cancer Chemother Pharmacol 60: 275-283, 2007.

12. Piacentini P, Donadelli M, Costanzo C, et al: Trichostatin A enhances the response of chemotherapeutic agents in inhibiting pancreatic cancer cell proliferation. Virchows Arch 448: 797-804, 2006.

13. Zhang X, Yashiro M, Ren J and Hirakawa K: Histone deacetylase inhibitor, trichostatin A, increases the chemosensitivity of anticancer drugs in gastric cancer cell lines. Oncol Rep 16: 563-568, 2006.

14. Glaser KB: HDAC inhibitors: clinical update and mechanismbased potential. Biochem Pharmacol 74: 659-671, 2007.

15. Munshi A, Tanaka T, Hobbs ML, et al: Vorinostat, a histone deacetylase inhibitor, enhances the response of human tumor cells to ionizing radiation through prolongation of gamma-H2AX foci. Mol Cancer Ther 5: 1967-1974, 2006.

16. Takemura H, Rao VA, Sordet O, et al: Defective Mre11dependent activation of Chk2 by ataxia telangiectasia mutated in colorectal carcinoma cells in response to replication-dependent DNA double strand breaks. J Biol Chem 281: 30814-30823, 2006.

17. Chinnaiyan P, Chowdhary S, Brem S, et al: A Phase I trial of vorinostat in combination with bevacizumab and irinotecan in recurrent glioblastoma. Merk, NCT00762255, 2009.

18. Flis S, Gnyszka A and Splawinski J: HDAC inhibitors, MS275 and SBHA, enhances cytotoxicity induced by oxaliplatin in the colorectal cancer cell lines. Biochem Biophys Res Commun 387: 336-341, 2009.

19. Todd RC and Lippard SJ: Inhibition of transcription by platinum antitumor compounds. Metallomics 1: 280-291, 2009.

20. Glaser KB, Staver MJ, Waring JF, et al: Gene expression profiling of multiple histone deacetylase (HDAC) inhibitors: defining a common gene set produced by HDAC inhibition in T24 and MDA carcinoma cell lines. Mol Cancer Ther 2: 151-163, 2003.

21. Lee JH, Park JH, Jung Y, et al: Histone deacetylase inhibitor enhances 5-fluorouracil cytotoxicity by down-regulating thymidylate synthase in human cancer cells. Mol Cancer Ther 5: 3085-3095, 2006. 